\title{
Improvement of a Circular Microstrip Antenna Excited by Four Feeds and Suspended with Artificial Magnetic Conductors
}

\author{
Sanchai Eardprab, ${ }^{1}$ Chuwong Phongcharoenpanich, ${ }^{1}$ and Danai Torrungrueng ${ }^{2}$ \\ ${ }^{1}$ Faculty of Engineering, King Mongkut's Institute of Technology Ladkrabang, Bangkok 10520, Thailand \\ ${ }^{2}$ Faculty of Engineering and Technology, Asian University, Chon Buri 20260, Thailand \\ Correspondence should be addressed to Chuwong Phongcharoenpanich; pchuwong@gmail.com
}

Received 18 March 2013; Accepted 4 July 2013

Academic Editor: Francisco Falcone

Copyright (c) 2013 Sanchai Eardprab et al. This is an open access article distributed under the Creative Commons Attribution License, which permits unrestricted use, distribution, and reproduction in any medium, provided the original work is properly cited.

\begin{abstract}
The proposed antenna is a circular microstrip structure excited by four feeds and suspended with artificial magnetic conductors (AMCs). The multifeed circular microstrip antennas can generate a high circularly polarized performance by using a different feed arrangement. AMC structures with a square, circular, or octagonal patch on a unit cell are designed and applied to circular microstrip patch antennas for the enhancement of antenna performance. It is found that simulated results of the proposed antenna are well suited. The properties of wide beamwidth with good axial ratio can be achieved when applying the proposed AMC structures to circular microstrip antennas. The antenna prototype was fabricated to validate simulated results.
\end{abstract}

\section{Introduction}

Circularly polarized antennas are particularly interesting for many wireless communication applications, where their typical applications vary from wireless LAN, satellite communications, and automotive applications, especially on applications in multipath and fading environment. The advantage of circularly polarized antennas is that specific antenna orientation and good immunity of signals in the case of multipath propagation are not required. One type of widely used circularly polarized antennas is microstrip patch antennas because they are of light weight, low profile and low cost. However, microstrip patch antennas are narrowband and wide-beam antennas with narrow impedance and axial ratio bandwidths.

There are many techniques that make microstrip patch antennas generate a circular polarization. One of them is a multi-feed arrangement technique for circular microstrip patch antennas. From the literature in [1], it was shown that circular microstrip patch antennas can provide circularly polarized characteristics by using different feed arrangement. In addition, this technique can preserve beam symmetry and decrease axial ratio. The finite ground plane effect was also studied in that literature. The results indicate that a ground plane size of $3 \lambda \times 3 \lambda$ is quite good despite the inclusion of finite ground plane effect, where $\lambda$ is the wavelength in free space. It makes the overall size of the antenna larger for above applications. On the other hand, the radiation efficiency is lower, caused by an aperture loss from larger patches, leading to lower antenna gain.

The reflector surface structure is one useful technique that has been employed to enhance the performance of microstrip patch antennas such as electromagnetic band gap (EBG) structures $[2,3]$. AMCs with several shapes of patch on a unit cell, such as rectangular shape $[4,5]$, modified rectangular shape $[6-8]$, spiral shape $[9,10]$, and Jerusalem cross shape [11], have been studied to solve microstrip patch antenna problems. In comparison between EBG and AMC structures [12], EBGs are periodic substrates which include small patches on a substrate with connecting vias between the patches and the ground plane. It provides high impedance within a certain frequency range and in-phase reflection characteristics for the incident wave. On the other hand, there are no vias between the patches and the ground plane in AMC structures, but the in-phase reflection behaviour remains. The AMC structure applications have been studied in [4-17], and it is realized that AMC structures can enhance the antenna performance. 
Therefore, in this work, an application of AMC structures with several shapes of patch on a unit cell to the circular microstrip antenna excited by four feeds is proposed. The square, circular, and octagonal shapes of patch on a unit cell are investigated to enhance the antenna performance, especially bandwidth and antenna gain. It is found that simulated results of the proposed antenna are well suited for the target specifications with improvement of the bandwidth and antenna gain, especially the antenna with the AMC structure of an octagonal shape of patch on a unit cell. The antenna prototype was fabricated to validate simulated results.

The organization of this paper is as follows. The analysis of circular microstrip antennas is briefly discussed in Section 2. Section 3 provides the design of circular microstrip antennas with AMC structures. The antenna prototype and measured results are discussed in Section 4. Finally, conclusions are given in Section 5.

\section{Brief Analysis of Circular Microstrip Antennas}

For a typical circular microstrip antenna [1], its lowest mode is the $\mathrm{TM}_{11}$. The fundamental mode can only generate a broadside and linearly polarized radiation. In order to achieve circular polarization characteristics, multiple feeds with proper angular separation are required. Figure 1 illustrates the geometry of the circular microstrip antenna, where $d$ is the diameter of the circular patch, $D$ is a diameter of the circular substrate, $G_{p}$ is the size of the square ground plane, and $h$ is the thickness of the substrate with the dielectric constant of $\varepsilon_{r}$. Notice that the geometry of the substrate is a circular shape in order to enhance the radiation symmetry, and the ground plane is suspended with an air gap of $H_{\text {air }}$ between the circular microstrip patch antenna and the ground plane.

The resonant frequency of a circular microstrip radiator for any mode can be determined as [18]

$$
f_{n m}=\frac{X_{n m} c}{2 \pi a_{e} \sqrt{\varepsilon_{r}}}
$$

where $c$ is the speed of light, $\varepsilon_{r}$ is the dielectric constant, $a_{e}$ is the effective radius of the patch, and $X_{n m}$ is $m$ th zero of the derivative of the Bessel function of order $n$. Note that $n$ represents the angular mode, while $m$ represents the radial mode. Some useful values of $X_{n m}$ are given in Table 1 .

The circular polarization mechanism by using the multifeed excitation can be described as follows [1]. The two feeds with a proper angular separation are needed. With the spacing, the fields generated from the two feeds are orthogonal to each other under the patch as well as outside the patch. In addition, with this angular spacing, one probe is always situated in the null field region of the other probe, thus, causing very little mutual coupling between the probes. Certainly, as is conventionally done, the two probes are required to be fed $90^{\circ}$ out of phase for achieving circular polarization. The angular spacing between two feed probes is different for each mode. In order to preserve beam symmetry and to keep crosspolarization low, especially for relatively thick substrate, the unwanted modes need to be suppressed. Generally, the two
TABLE 1: Useful values of $X_{n m}$.

\begin{tabular}{llllll}
\hline & $\mathrm{TM}_{11}$ & $\mathrm{TM}_{21}$ & $\mathrm{TM}_{31}$ & $\mathrm{TM}_{41}$ & $\mathrm{TM}_{51}$ \\
\hline$X_{n m}$ & 1.841 & 3.054 & 4.201 & 5.317 & 6.415 \\
\hline
\end{tabular}

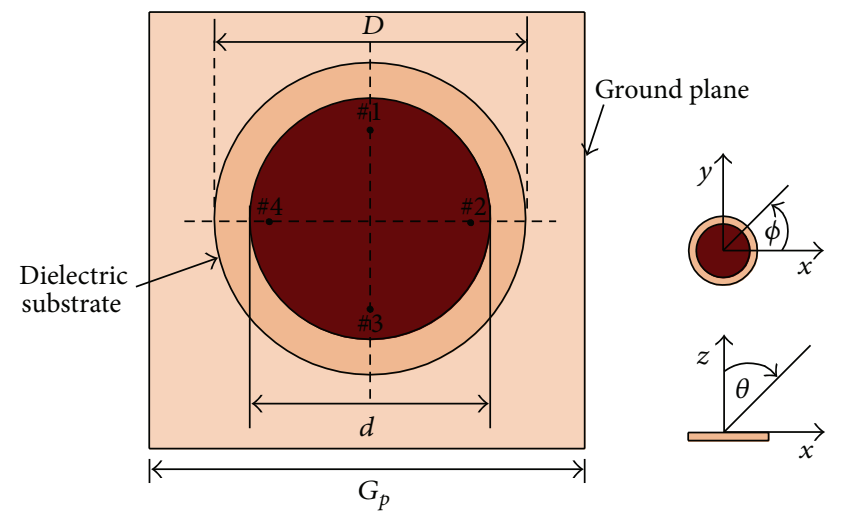

(a)

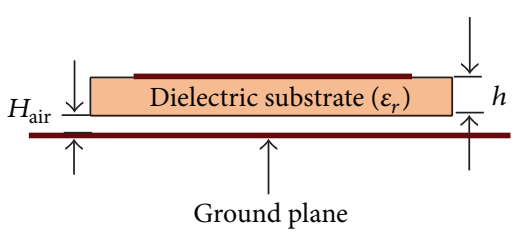

(b)

Figure 1: Circular microstrip antenna geometry. (a) Top view. (b) Side view.

neighboring modes of a resonant mode have the next highest magnitudes. One way to suppress these adjacent modes is to employ two additional feed probes located diametrically across from the two original feeds. Together, these four feeds should have a phase arrangement of $0^{\circ}, 90^{\circ}, 180^{\circ}$, and $270^{\circ}$ for the odd-order modes so that the fields of the unwanted modes from the two opposing feeds cancel. In addition, for this configuration of the antenna from Figure 1, the lefthand circular polarization (LHCP) or right-hand circular polarization (RHCP) can be achieved by using proper phase arrangement for each feed point, that is, for LHCP using $0^{\circ}$, $90^{\circ}, 180^{\circ}$, and $270^{\circ}$ and for RHCP using $270^{\circ}, 180^{\circ}, 90^{\circ}$, and $0^{\circ}$.

The effect of substrate of microstrip antenna has been studied [18-20]. The parallel-plate effect between the patch and the ground plane causes the fringing field to extend beyond the edge of the patch. This increases the capacitance between the patch and the ground plane, resulting in the lower resonant frequency. From the experimental study of microstrip antennas on substrate thickness [18], it results in changing of resonant frequency, bandwidth, and impedance of the antenna. For the effect of the substrate permittivity, the higher substrate permittivity reduces the patch size and the size of the radiation zone [20]. In order to preserve beam symmetry and decrease axial ratio, the finite ground plane effect [21-24] is considered. In practice, a large ground plane is not realistic; therefore, practical antenna ground plane dimensions must be finite with small size as possible. Therefore, the ground plane of the antenna in this work will be small finite size suitable for antenna requirements. 
In addition, an antenna with suspended ground plane can enhance impedance and radiation performance that has been investigated in [25]. From the previous brief analysis, it will be employed to design the circular microstrip patch antenna with AMC structures in Section 3.

\section{Design of Circular Microstrip Antennas with AMC Structures}

In this section the AMC structure with several shapes of patch on a unit cell is designed and applied to the circular microstrip patch antenna for enhancement of the antenna performance. The antenna requirements in Table 2 are employed as a guideline in this design work. The operating frequency range of wireless LAN application is used. The antenna dimension is designed to be compact and suitable for several applications. The substrate material is FR-4 because it is not expensive and widely used in electronic industry. The radiation characteristics of antenna with unidirectional radiation pattern, circular polarization, and antenna gain of over $5 \mathrm{dBic}$ are required.

3.1. Artificial Magnetic Conductor. The AMC is one of metamaterials exhibiting novel electromagnetic features [4-17], where its structure has a specific property regarding its reflection phase. It is known that a perfect electric conductor (PEC) has a $180^{\circ}$ reflection phase for a normal incident wave, while a perfect magnetic conductor (PMC), which does not exist in nature, has a $0^{\circ}$ reflection phase. AMCs exhibit $0^{\circ}$ reflection phase like PMCs at the resonant frequency [2]. Another property of AMCs is high surface impedance that can suppress surface waves when AMCs are used as a reflector of antennas. Suppression of surface waves results in increased antenna gain and reduced back lobe level compared to common metal reflectors [17].

In this section, AMC structures will be designed with the operating frequency of $2.45 \mathrm{GHz}$. Figures 2(a) and 2(b) show schematic diagrams of the AMC structure with a square patch in a unit cell, where $W_{\text {pa }}$ and $W_{\text {gap }}$ are the patch width and the gap width, respectively. It is designed on substrates with a thickness of $H_{\mathrm{amc}}$ and relative permittivity $\varepsilon_{r}$ of 4.3 (FR-4 substrate). The equivalent circuit representation of the AMC structure with a square patch in a unit cell is shown in Figure 2(c), where the conducting element is represented by the inductor $L$ and the interelement capacitance by the capacitor $C$ [5]. Figure 2(d) shows the CST MICROWAVE STUDIO [26] simulation model applied from [4]. It is initially used to provide guidelines on the AMC design in this work. The bandwidth of the AMC structure with a square patch is studied by using the reflection phase. The useful bandwidth of AMCs in a unit cell is defined as $+90^{\circ}$ to $-90^{\circ}$ on either side of the resonant frequency since these phase values would not cause destructive interference between direct and reflected waves [7]. The effect of the substrate thickness of AMCs on the reflection phase is shown in Figure 3. It is found that the higher substrate thickness of AMCs provides the range of the bandwidth of this AMC structure performance wider than the lower one. The useful bandwidths of AMC are $3.22 \%$,
TABLE 2: The antenna requirements.

\begin{tabular}{lc}
\hline Parameter & Value \\
\hline Frequency range & $2400-2484 \mathrm{MHz}($ Wireless LAN $)$ \\
Dimension (maximum) & $100 \mathrm{~mm} \times 100 \mathrm{~mm} \times 10 \mathrm{~mm}$ \\
Material & FR- 4 \\
Radiation type & Unidirectional \\
Polarization & LHCP or RHCP \\
Gain & $>5 \mathrm{dBic}$ \\
\hline
\end{tabular}

$6.76 \%, 14.45 \%$, and $19.71 \%$, when the substrate thicknesses are $0.8 \mathrm{~mm}, 1.5 \mathrm{~mm}, 3.0 \mathrm{~mm}$, and $4.5 \mathrm{~mm}$, respectively.

For the next step, the other shapes of patch on a unit cell are investigated. Figure 4 illustrates the schematic diagrams of the two types of AMC patches, where Figure 4(a) is the unit cell of circular patch with the diameter of $W_{\mathrm{pa}}$ and Figure $4(\mathrm{~b})$ is the unit cell of octagonal patch with the length of a side of regular octagon of $W_{\text {pa }}$. The parametric study of the patch width and the gap width of AMC structures with three types of AMC patches is performed in Figure 5. It is found that the curves of the patch width versus the gap width tend to converge when the gap width is increased. By using the same substrate thickness of AMC structures, it is found that the AMC unit cell with a square patch provides the patch size smaller than the others, while the size of the circular patch is the largest. On the other hand, the higher substrate thickness of AMC structures provides the patch size smaller than the lower one. Finally, the results in Figure 5 are useful in design of circular microstrip antennas with AMC structures in the next section.

\subsection{Circular Microstrip Patch Antennas with AMC Structures.} In this section, AMC structures with three patch shapes are designed and applied to circular microstrip patch antennas excited by four feeds for the enhancement of antenna performance. Based on the analysis in the previous section, the resonant frequency of a circular microstrip at the operating frequency is controlled by two parameters from (1), that is, the radius of the radiating patch and the dielectric constant of the substrate. Once the dielectric constant of substrate is known, the radius of the radiating patch can be estimated by using (1). In the next step, results in Figure 5 are considered by properly selecting the substrate thickness and the gap width of AMC structures of interest. Therefore, three AMC patch shapes with the substrate dielectric constant of $4.3, W_{\mathrm{pa}}$ of $3 \mathrm{~mm}$, and $W_{\text {gap }}$ of $1.25 \mathrm{~mm}$ will be applied to design a circular microstrip patch antenna with $h$ of $1.5 \mathrm{~mm}$ and $H_{\text {air }}$ of $1.5 \mathrm{~mm}$. The geometry of the circular microstrip patch antenna with three AMC structures is shown in Figure 6. The ground plane of microstrip patch antenna in Figure 1 is replaced with the AMC structure of $3 \times 3$ unit cells, where the model-S, model$\mathrm{C}$, and model-O are the circular microstrip patch antenna of the AMC structure with square, circular, and octagonal patches, respectively.

The reflection characteristics of the antenna with the AMC structures are shown in Figure 7, where the reflection coefficients at each port $\left(\left|S_{11}\right|,\left|S_{22}\right|,\left|S_{33}\right|\right.$, and $\left.\left|S_{44}\right|\right)$ are identical due to the symmetry of the feed position. It is 


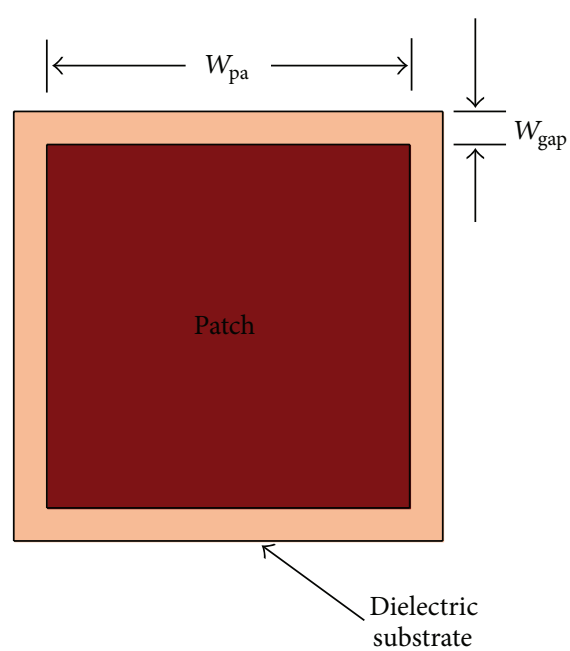

(a)

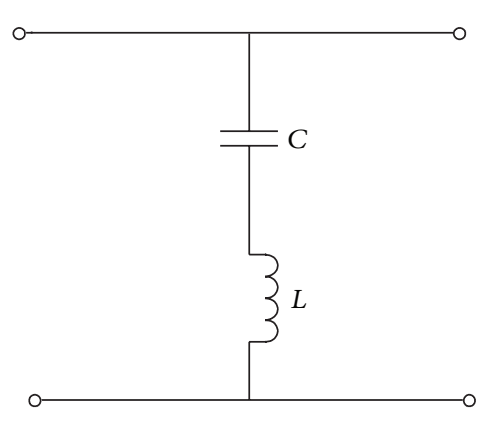

(c)

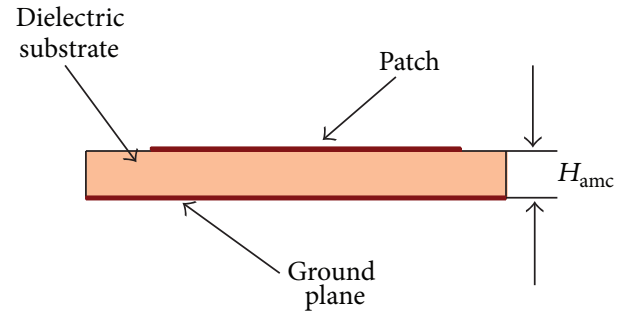

(b)

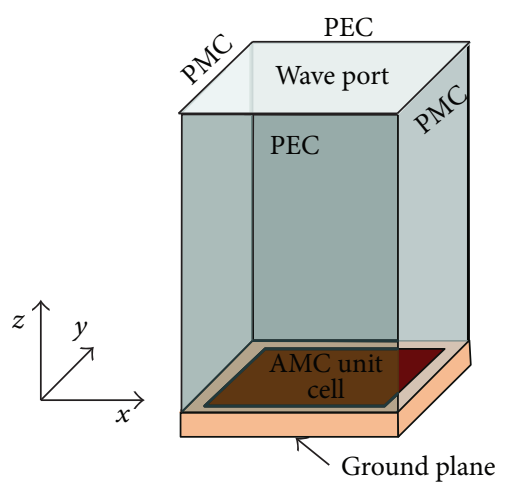

(d)

Figure 2: A unit cell of AMC structures with a square patch. (a) Front view. (b) Side view. (c) Equivalent circuit. (d) Simulation model.

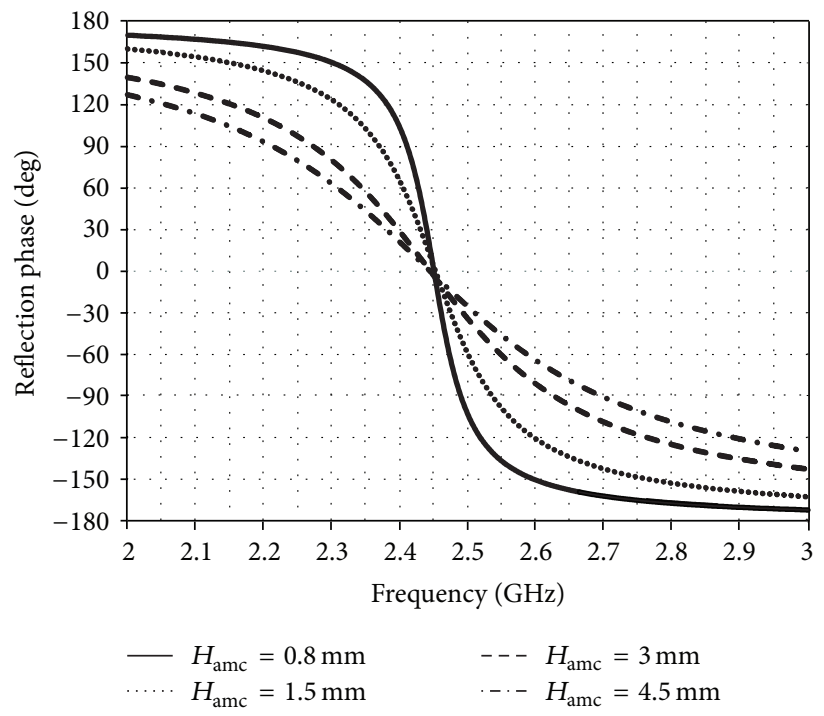

FIgURE 3: The reflection phase characteristics of the AMC structure with a square patch in a unit cell with different thickness of dielectric substrate, where $W_{\text {gap }}=1.25 \mathrm{~mm}$ and the values of $W_{\mathrm{pa}}$ are $27.38 \mathrm{~mm}, 25.6 \mathrm{~mm}, 21.25 \mathrm{~mm}$, and $17.15 \mathrm{~mm}$, for $H_{\mathrm{amc}}$ of $0.8 \mathrm{~mm}, 1.5 \mathrm{~mm}, 3.0 \mathrm{~mm}$, and $4.5 \mathrm{~mm}$, respectively. 


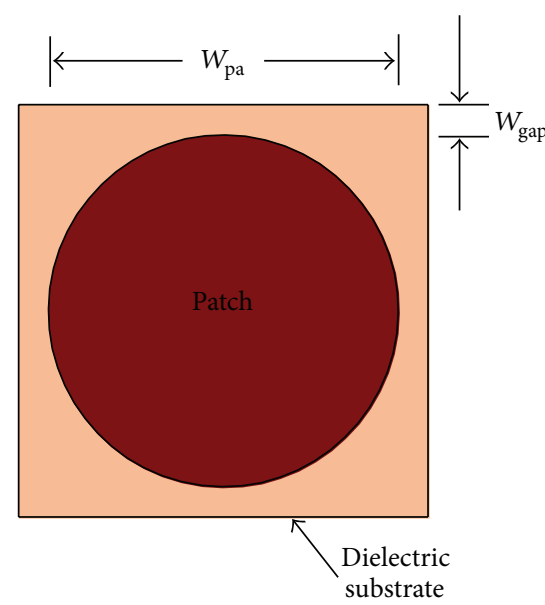

(a)

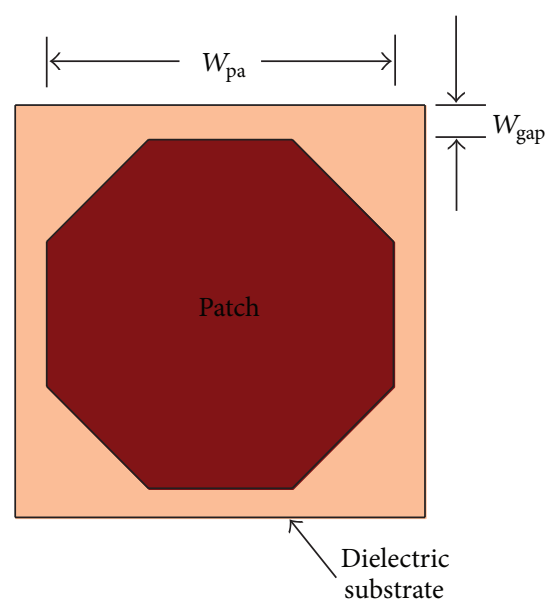

(b)

Figure 4: A unit cell of an AMC structure. (a) A circular patch. (b) An octagonal patch.

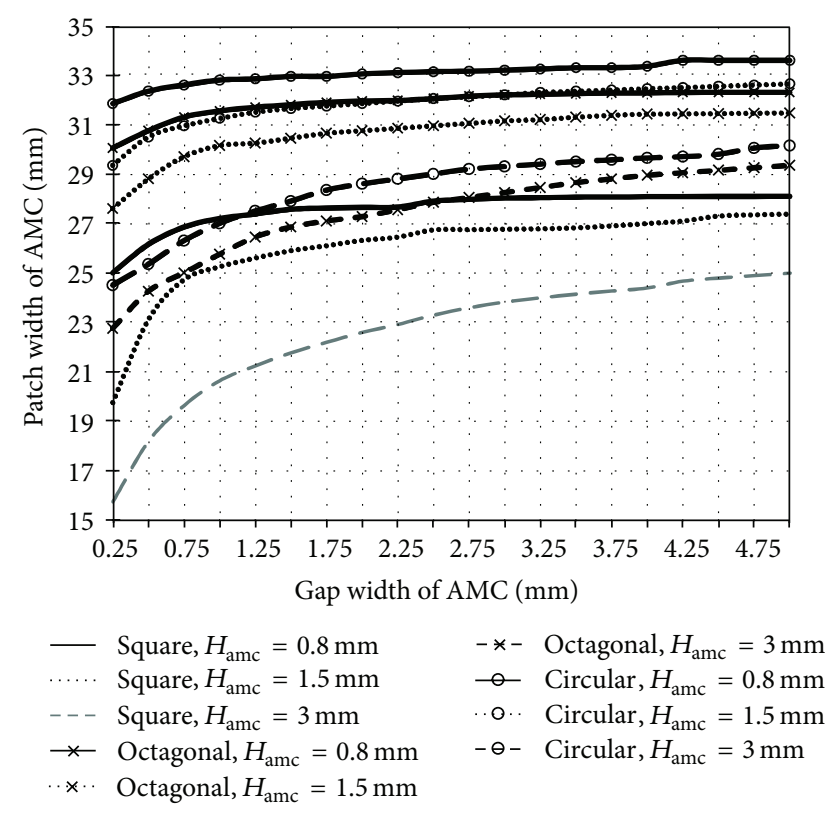

FIGURE 5: The parametric study of the patch width and the gap width of AMC structures at the operating frequency of $2.45 \mathrm{GHz}$.

found that the presence of the AMC structures leads to wider impedance bandwidths $\left(\left|S_{\mathrm{ii}}\right|\right.$ is less than $\left.-10 \mathrm{~dB}\right)$ of $11.63 \%$, $17.76 \%$ and $17.14 \%$ for the model-S, model-C, and model$\mathrm{O}$, respectively, at the operating frequency of $2.45 \mathrm{GHz}$, in comparison to $8.67 \%$ for the reference antenna. The radiation characteristics of the circular microstrip antenna with the AMC structures are shown in Figure 8. It is found that the presence of the AMC structures can reduce the back lobe and improve the axial ratio beamwidth, although it makes the directivity decrease. The LHCP or RHCP can be achieved by using proper phase arrangement for each feed point as described in Section 2. In addition, for comparison of the axial ratio versus frequency, the axial ratios are identical and equal to $0 \mathrm{~dB}$ covering over $2-3 \mathrm{GHz}$ due to the advantage of the multi-feed arrangement technique.

The summary of simulated results of the circular microstrip antenna with AMC structures at the operating frequency of $2.45 \mathrm{GHz}$ is tabulated in Table 3. It is clear that the presence of the AMC structures can enhance the antenna performance, especially the impedance bandwidth and antenna gain. In comparison among the AMC structures, the circular microstrip antenna with the AMC model-S has the maximum dimension smaller than the other models, but its antenna performance is the lowest. On the other hand, the circular microstrip antenna with the AMC model$\mathrm{C}$ has the best antenna performance, but it has the largest maximum dimension. The circular microstrip antenna with the AMC model-O has the antenna performance similar to the AMC model-C, but its maximum dimension is smaller. Therefore, the circular microstrip antenna with the AMC model-O is selected as the antenna prototype in order to validate simulated results in Section 4.

\section{Antenna Prototype and Measurement}

In this section, the prototype of the circular microstrip antenna with the AMC model-O was fabricated. The parameters of the circular microstrip antenna are defined as follows: $d=0.325 \lambda, D=1.65 d$, and $h=1.5 \mathrm{~mm}$, where $\lambda$ is equal to $122.45 \mathrm{~mm}$. All feed points are excited with the $\mathrm{TM}_{11}$ mode using a proper phase arrangement for each feed point $\left(0^{\circ}, 90^{\circ}, 180^{\circ}\right.$, and $\left.270^{\circ}\right)$ to achieve LHCP for illustration purpose and the distances from the center of the patch to all feed points are $0.45 d$. The AMC model-O of $3 \times 3$ unit cells possesses the total size of $83.10 \mathrm{~mm} \times 83.10 \mathrm{~mm}$. The photograph of the fabricated antenna prototype and its impedance characteristics are shown in Figure 9. From Figure 9(a), it shows the front and bottom views of the antenna prototype. Note that the four SMA-connectors are employed to excite the antenna prototype as shown in the bottom view. From the S-parameter characteristics in Figure $9(\mathrm{~b})$, it is found 


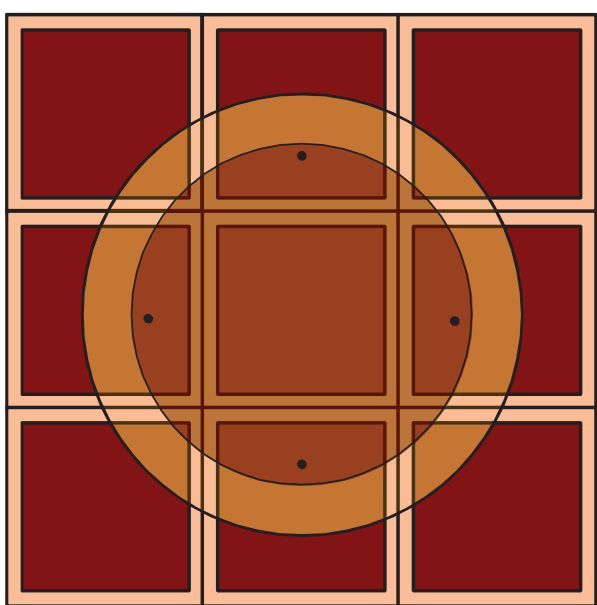

(a)

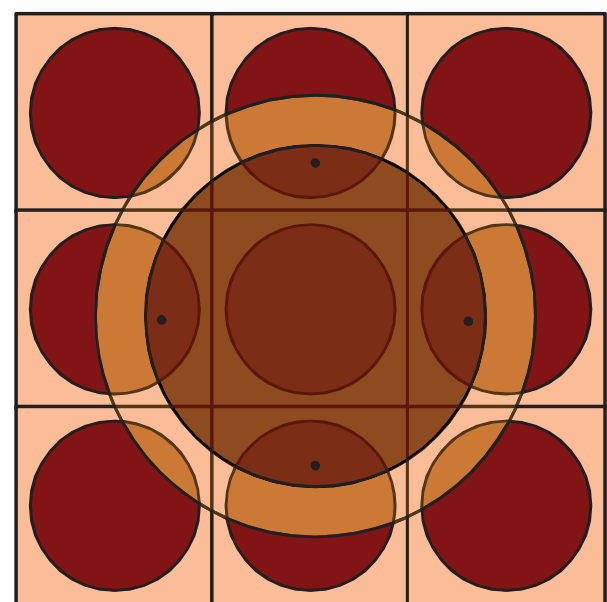

(b)

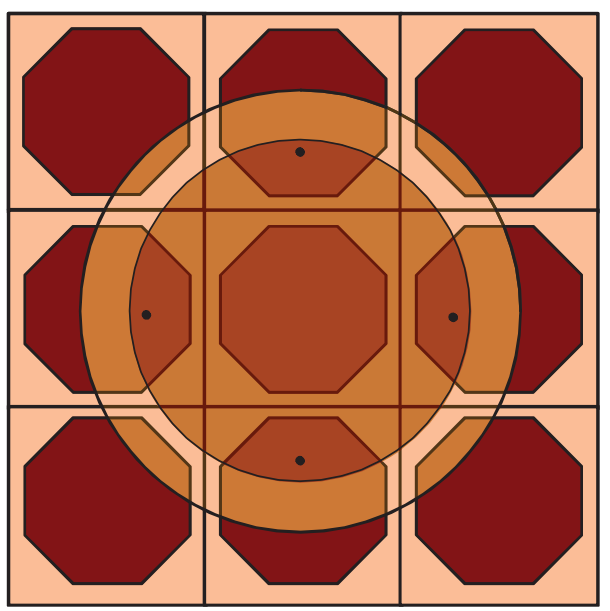

(c)

FIgure 6: The geometry of the circular microstrip patch antenna with AMC structures. (a) Model-S. (b) Model-C. (c) Model-O.

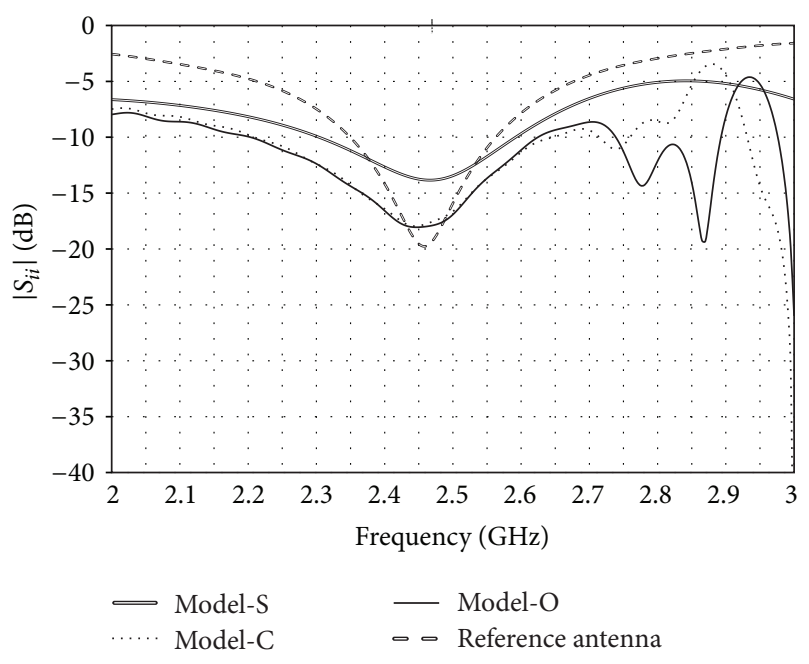

FIgURE 7: The reflection characteristics of the circular microstrip patch antenna with the AMC structure. that the trend of the measured S-parameters of each port reasonably agrees with simulated results. At the $2.45 \mathrm{GHz}$ operating frequency, $\left|S_{11}\right|,\left|S_{22}\right|,\left|S_{33}\right|$, and $\left|S_{44}\right|$ are lower than $-10 \mathrm{~dB}$ covering the bandwidth for the target application, although the center frequency of bandwidth is shifted down to around $2.3 \mathrm{GHz}$. This may be due to the fact that the feed points on the antenna structure are not identical in fabrication.

On the other hand, Figure 10 illustrates the measured radiation characteristics of the circular microstrip antenna with the AMC model-O. The measured radiation pattern in Figure 10(a) is reasonably in good agreement with the simulated ones, especially in the main lobe. Similarly, the measured and simulated axial ratios in Figure 10(b) have similar trend. It is noticed that the radiation pattern and axial ratio are not symmetry, especially in the back lobe, caused by the existence of SMA-connectors and coaxial lines, on the back of the antenna for the measurement.

Figure 11 shows the measured gain and axial ratio at $\theta=0^{\circ}$ as a function of frequency. In Figure 11(a), it is 
TABLE 3: Simulated results of the circular microstrip antenna with AMC structures at the operating frequency of $2.45 \mathrm{GHz}$.

\begin{tabular}{|c|c|c|c|c|}
\hline Model & Model-S & Model-C & Model-O & Reference antenna \\
\hline Maximum dimension (mm) & 67.50 & 86.25 & 83.10 & 90.00 \\
\hline Diameter of the patch of the microstrip antenna (mm) & 36.12 & 41.63 & 39.80 & 55.71 \\
\hline Impedance bandwidth (\%) & 11.63 & 17.76 & 17.14 & 8.67 \\
\hline Directivity $(\mathrm{dBi})$ & 7.479 & 8.264 & 8.154 & 9.164 \\
\hline Gain $(\mathrm{dBic})$ & 6.818 & 7.332 & 7.317 & 6.045 \\
\hline Total efficiency $(\mathrm{dB})$ & -0.661 & -0.932 & -0.836 & -3.119 \\
\hline Half-power beamwidth (deg) & 80 & 70.6 & 71.7 & 64.9 \\
\hline Axial ratio beamwidth (deg) & 197.43 & 220.38 & 228.34 & 156.76 \\
\hline Polarization & \multicolumn{4}{|c|}{ LHCP or RHCP } \\
\hline
\end{tabular}

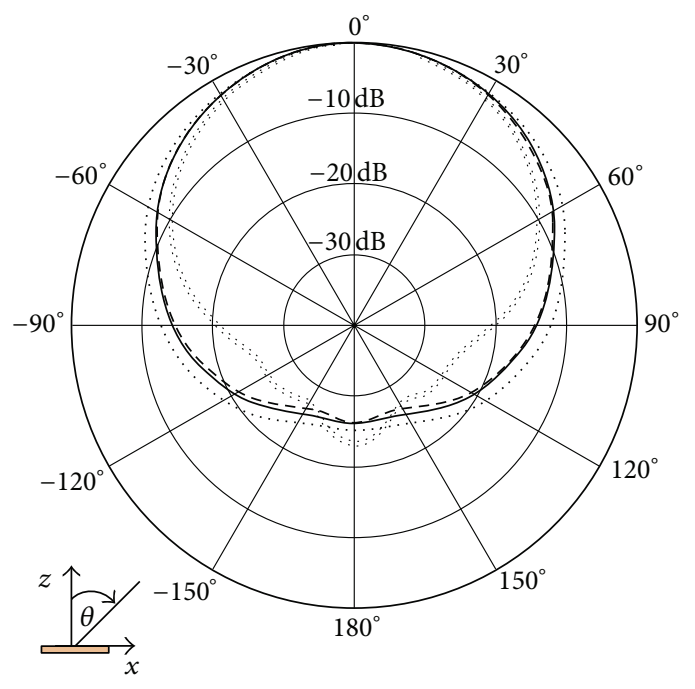

(a)

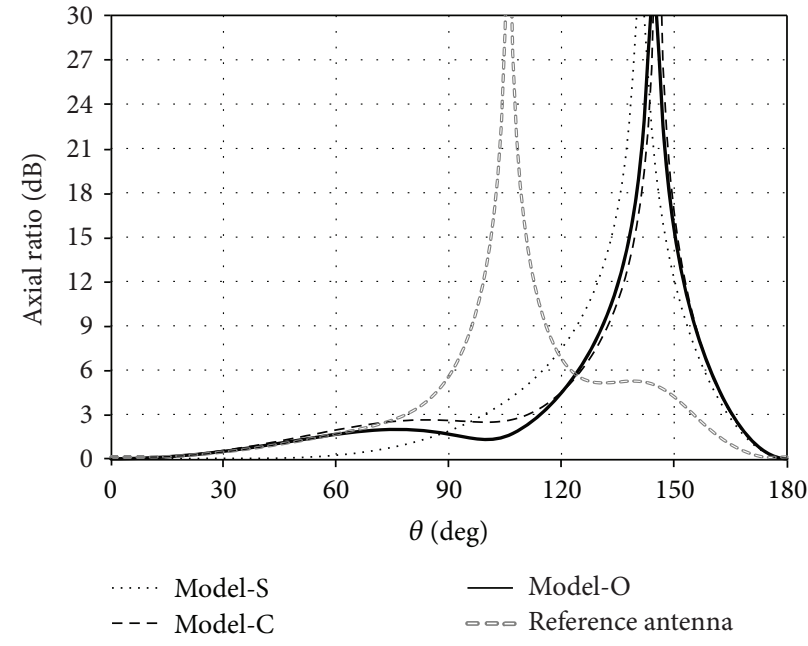

(b)

FIgURE 8: Simulated results of the radiation pattern and axial ratio of the circular microstrip antenna with AMC structures. (a) Radiation pattern. (b) Axial ratio.

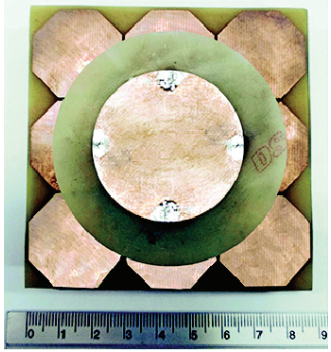

Top view

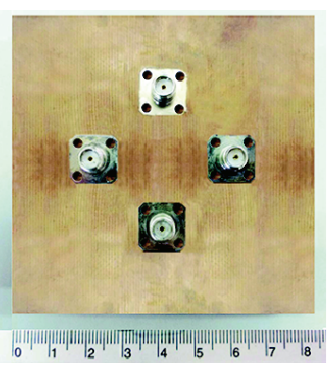

Bottom view

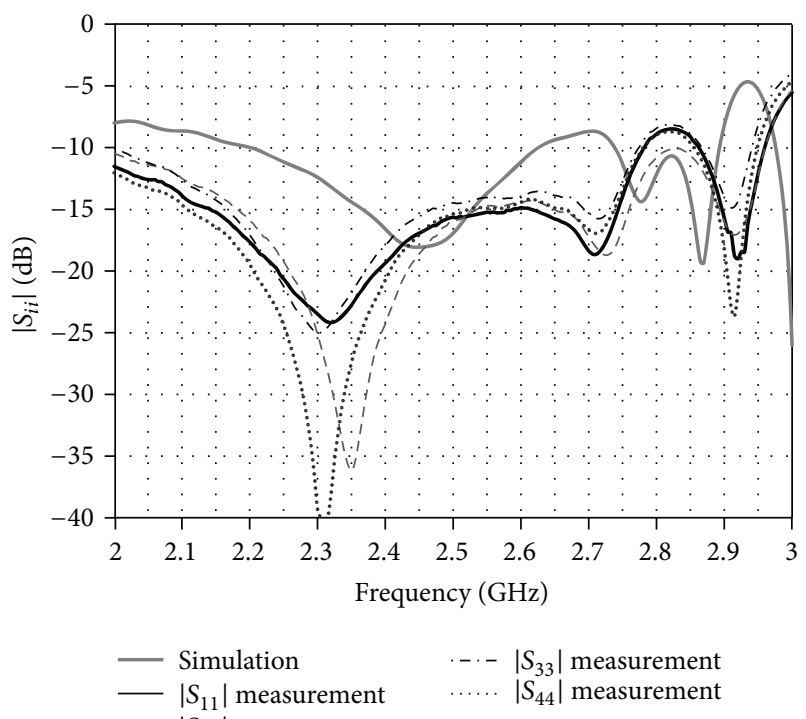

(a)

(b)

FIGURE 9: (a) Photograph of the antenna prototype. (b) The $S$ parameters as a function of frequency. 


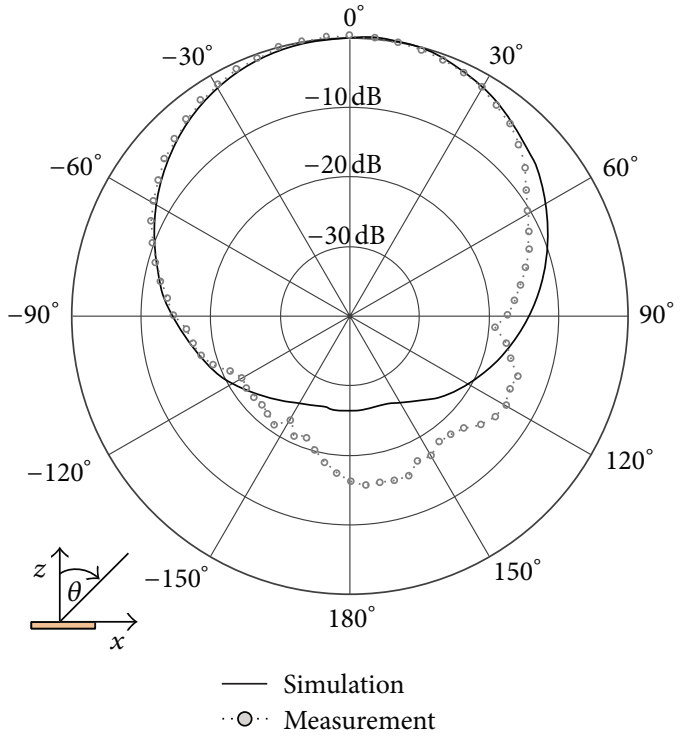

(a)

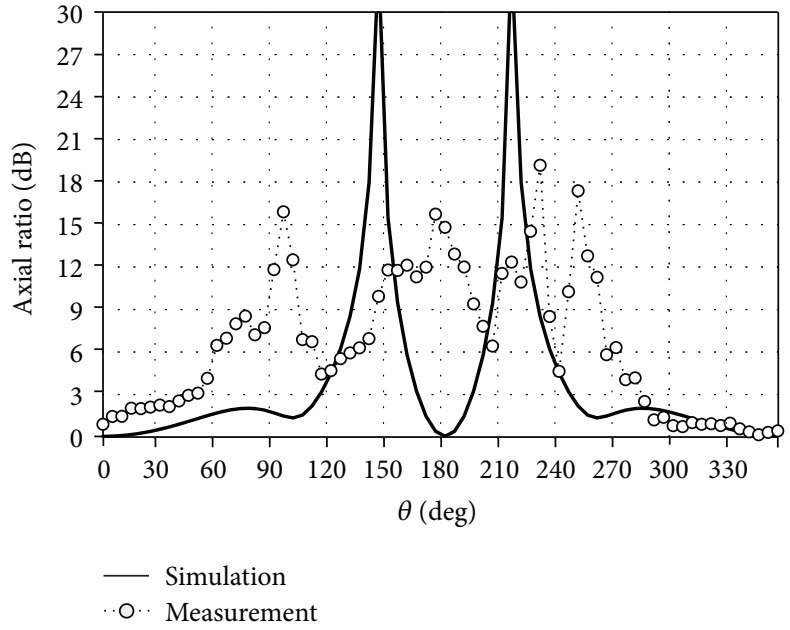

(b)

FIGURE 10: The measured radiation pattern and axial ratio of the circular microstrip antenna with the AMC model-O. (a) Radiation pattern. (b) Axial ratio.

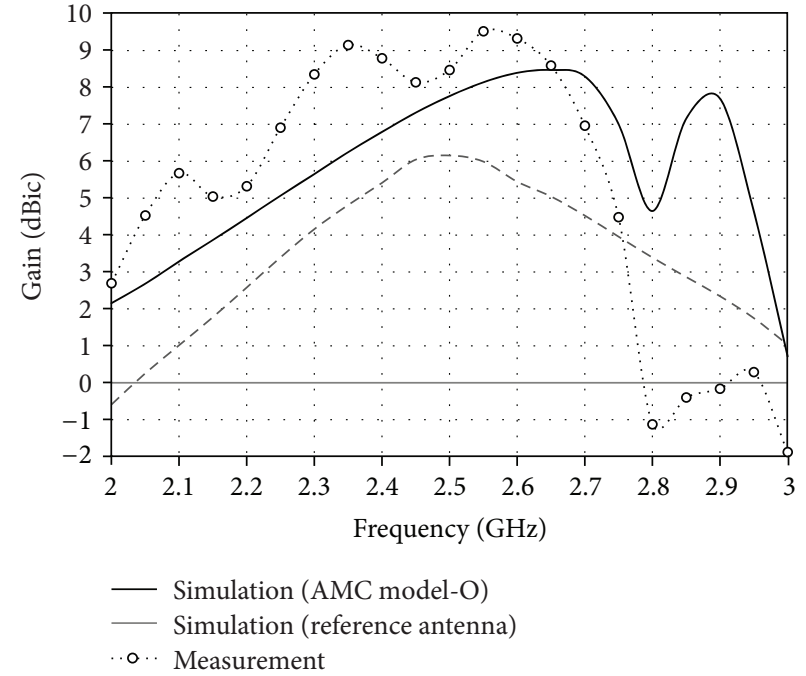

(a)

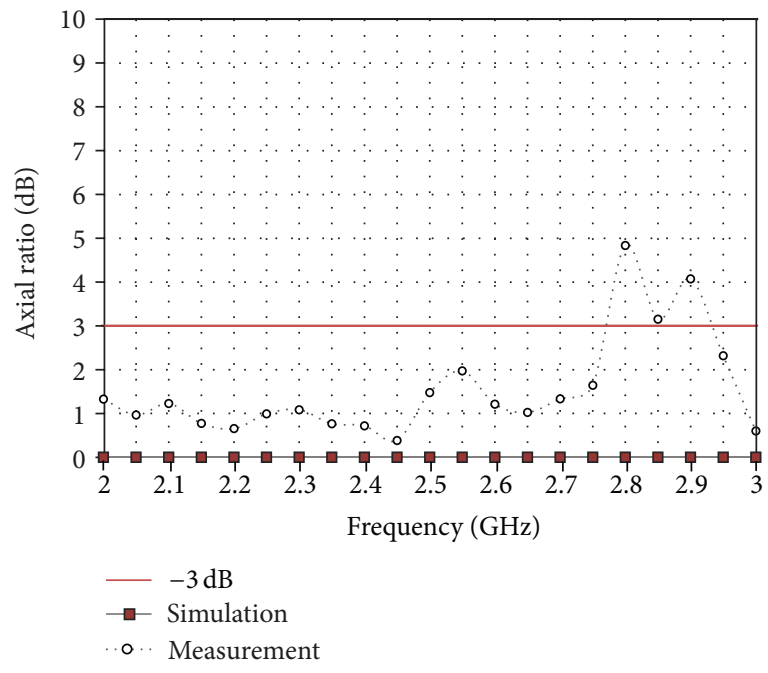

(b)

FIgURE 11: The measured gain and axial ratio as a function of frequency. (a) Gain. (b) Axial ratio.

found that the measured and simulated gains of the proposed antenna have similar trend in the frequency range of 2$3 \mathrm{GHz}$. Although the measured and simulated results have similar trend, the difference may be due to fabrication error of the antenna prototype and the measurement environment. The measured gain at $2.45 \mathrm{GHz}$ is $8.14 \mathrm{dBic}$ with reasonably good bandwidth. This high gain is due to the presence of the high AMC surface impedance, resulting in a suppression of surface waves, increased antenna gain, and reduced back lobe level [17]. In addition, the measured axial ratio as a function of frequency is shown in Figure 11(b). It is found that the measured axial ratio is mostly less than $3 \mathrm{~dB}$ in the frequency range of $2-3 \mathrm{GHz}$. Therefore, it is clear that the AMC structures can be used to enhance the antenna performance.

\section{Conclusions}

The proposed antenna is a circular microstrip antenna excited by four feeds and suspended with AMCs. Three AMC structures with square, circular, and octagonal patches are designed and applied to the circular microstrip patch antenna to enhance the antenna performance. In the AMC design, it is found that the larger substrate thickness of AMCs can provide 
the range of the bandwidth of AMC performance wider than the smaller one. In addition, it is found that the AMC structures can enhance the antenna performance, especially the impedance bandwidth and antenna gain. Among three AMC structures, the circular microstrip antenna with the AMC model-S is the most compact, but its antenna performance is the worst. On the other hand, the antenna with the AMC model-C provides the best antenna performance with the largest maximum dimension. However, the antenna with the AMC model-O compromises the formers and is employed in this study. Finally, the antenna prototype was fabricated to validate simulated results. It is found that the simulated and measured results possess similar trend. The development of this antenna will be performed in the future work, especially the feeding network and antenna performance for specified applications.

\section{Conflict of Interests}

This paper has supported the framework of a Ph.D. thesis. The authors declare that there is no conflict of interests with CST MICROWAVE STUDIO.

\section{Acknowledgment}

The financial support from CHE and AUN/SEED-Net Scholarship for this research is greatly acknowledged.

\section{References}

[1] J. Huang, "Circularly polarized conical patterns from circular microstrip antennas," IEEE Transactions on Antennas and Propagation, vol. 32, no. 9, pp. 991-994, 1984.

[2] F. Yang and Y. Rahmat-Samii, "A low profile single dipole antenna radiating circularly polarized waves," IEEE Transactions on Antennas and Propagation, vol. 53, no. 9, pp. 3083-3086, 2005.

[3] D. Qu, L. Shafai, and A. Foroozesh, "Improving microstrip patch antenna performance using EBG substrates," IEE Proceedings: Microwaves, Antennas and Propagation, vol. 153, no. 6, pp. 558$563,2006$.

[4] Y. Zhang, J. V. Hagen, M. Younis, C. Fischer, and W. Wiesbeck, "Planar artificial magnetic conductors and patch antennas," IEEE Transactions on Antennas and Propagation, vol. 51, no. 10 I, pp. 2704-2712, 2003.

[5] A. P. Feresidis, G. Goussetis, S. Wang, and J. C. Vardaxoglou, "Artificial magnetic conductor surfaces and their application to low-profile high-gain planar antennas," IEEE Transactions on Antennas and Propagation, vol. 53, no. 1 I, pp. 209-215, 2005.

[6] J. R. Sohn, K. Y. Kim, and H. S. Tae, "Comparative study on various artificial magnetic conductors for low-profile antenna," Progress in Electromagnetics Research, vol. 61, pp. 27-37, 2006.

[7] M. E. de Cos, Y. Álvarez, and F. Las-Heras, "A novel approach for RCS reduction using a combination of artificial magnetic conductors," Progress in Electromagnetics Research, vol. 107, pp. 147-159, 2010.

[8] A. Foroozesh and L. Shafai, "Investigation into the application of artificial magnetic conductors to bandwidth broadening, gain enhancement and beam shaping of low profile and conventional monopole antennas," IEEE Transactions on Antennas and Propagation, vol. 59, no. 1, pp. 4-20, 2011.
[9] Y. Kim, F. Yang, and A. Z. Elsherbeni, "Compact artificial magnetic conductor designs using planar square spiral geometries," Progress in Electromagnetics Research, vol. 77, pp. 43-54, 2007.

[10] D. N. Elsheakh, H. A. Elsadek, E. A. Abdallah, M. F. Iskander, and H. Elhenawy, "Ultrawide bandwidth umbrella-shaped microstrip monopole antenna using spiral artificial magnetic conductor (SAMC)," IEEE Antennas and Wireless Propagation Letters, vol. 8, pp. 1255-1258, 2009.

[11] M. Hosseini and M. Hakkak, "Characteristics estimation for jerusalem cross-based artificial magnetic conductors," IEEE Antennas and Wireless Propagation Letters, vol. 7, pp. 58-61, 2008.

[12] T. Nakamura and T. Fukusako, "Broadband design of circularly polarized microstrip patch antenna using artificial ground structure with rectangular unit cells," IEEE Transactions on Antennas and Propagation, vol. 59, no. 6, pp. 2103-2110, 2011.

[13] D. Kim and J. Yeo, "Low-profile RFID tag antenna using compact AMC substrate for metallic objects," IEEE Antennas and Wireless Propagation Letters, vol. 7, pp. 718-720, 2008.

[14] M. E. de Cos, F. L. Heras, and M. Franco, "Design of planar artificial magnetic conductor ground plane using frequencyselective surfaces for frequencies below $1 \mathrm{GHz}$," IEEE Antennas and Wireless Propagation Letters, vol. 8, pp. 951-954, 2009.

[15] E. Y. Kim, J. H. Yoon, Y. J. Yoon, and C. G. Kim, "Low profile dual-band reflector antenna with dual resonant AMC," in Proceedings of the IEEE International Symposium on Antennas and Propagation (APSURSI '11), pp. 1800-1803, usaSpokane, Wash, USA, July 2011.

[16] J. Baracco, L. Salghetti-Drioli, and P. de Maagt, "AMC low profile wideband reference antenna for GPS and GALILEO systems," IEEE Transactions on Antennas and Propagation, vol. 56, no. 8, pp. 2540-2547, 2008.

[17] J. Ahn, C. Son, K. Chang, and Y. Yoon, "The miniaturization and electrical performance improvement of base station array antenna with artificial magnetic conductor," in Proceedings of the IEEE International Symposium on Antennas and Propagation and USNC/URSI National Radio Science Meeting (APSURSI), pp. 1-4, June 2009.

[18] J. S. Dahele and K. F. Lee, "Effect of substrate thickness on the performance of a circular-disk microstrip antenna," IEEE Transactions on Antennas and Propagation, vol. 31, no. 2, pp. 358-360, 1983.

[19] A. K. Bhattacharyya and R. Garg, "Effect of substrate on the efficiency of an arbitrarily shaped microstrip patch antenna," IEEE Transactions on Antennas and Propagation, vol. 34, no. 10, pp. 1181-1188, 1986.

[20] J. R. James and P. S. Hall, Handbook of Microstrip Antennas, vol. 2, Peter Peregrinus, England, UK, 1988.

[21] M. Daginnus, R. Kronberger, and A. Stephan, "Ground plane effects on the performance of SDARS antennas," in Proceedings of the IEEE Antennas and Propagation Society International Symposium, pp. 748-751, June 2002.

[22] A. K. Bhattacharyya, "Effects of finite ground plane on the radiation characteristics of a circular patch antenna," IEEE Transactions on Antennas and Propagation, vol. 38, no. 2, pp. 152-159, 1990.

[23] A. A. Kishk and L. Shafai, "The effect of various parameters of circular microstrip antennas on their radiation efficiency and the mode excitation," IEEE Transactions on Antennas and Propagation, vol. 34, no. 8, pp. 969-976, 1986.

[24] V. Losada, R. R. Boix, and M. Horno, "Resonant modes of circular microstrip patches in multilayered substrates," IEEE 
Transactions on Microwave Theory and Techniques, vol. 47, no. 4, pp. 488-498, 1999.

[25] X. N. Low, Z. N. Chen, and W. K. Toh, "Ultrawideband suspended plate antenna with enhanced impedance and radiation performance," IEEE Transactions on Antennas and Propagation, vol. 56, no. 8, pp. 2490-2495, 2008.

[26] CST MICROWAVE STUDIO, http://www.cst.com. 

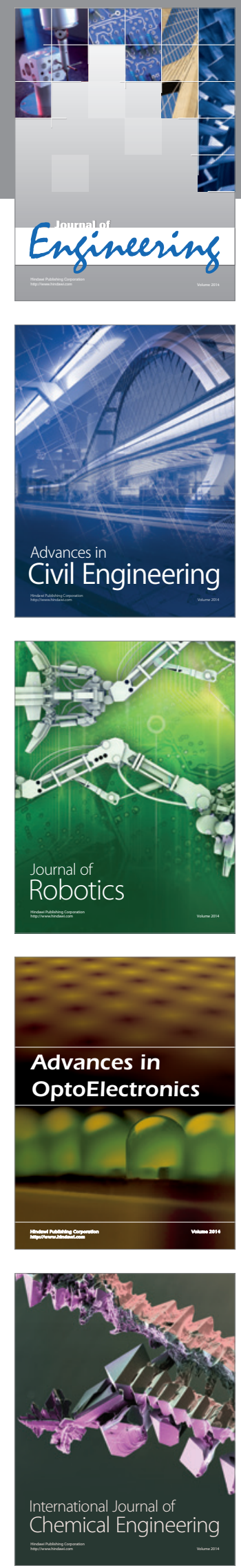

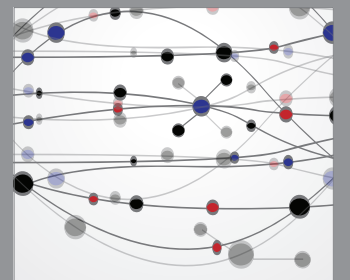

The Scientific World Journal
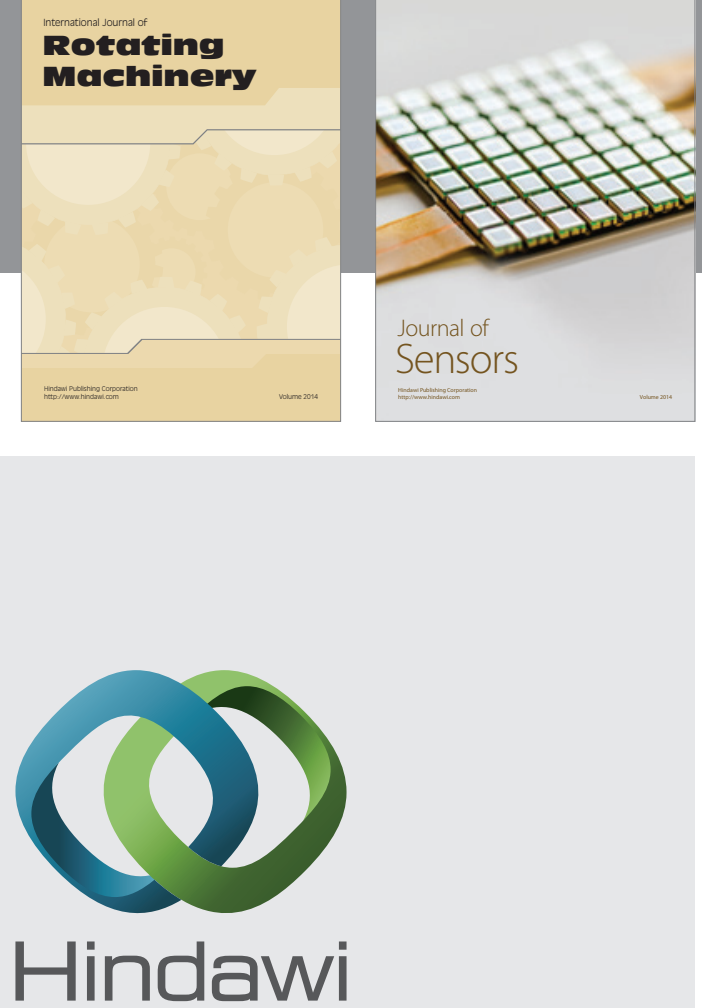

Submit your manuscripts at http://www.hindawi.com
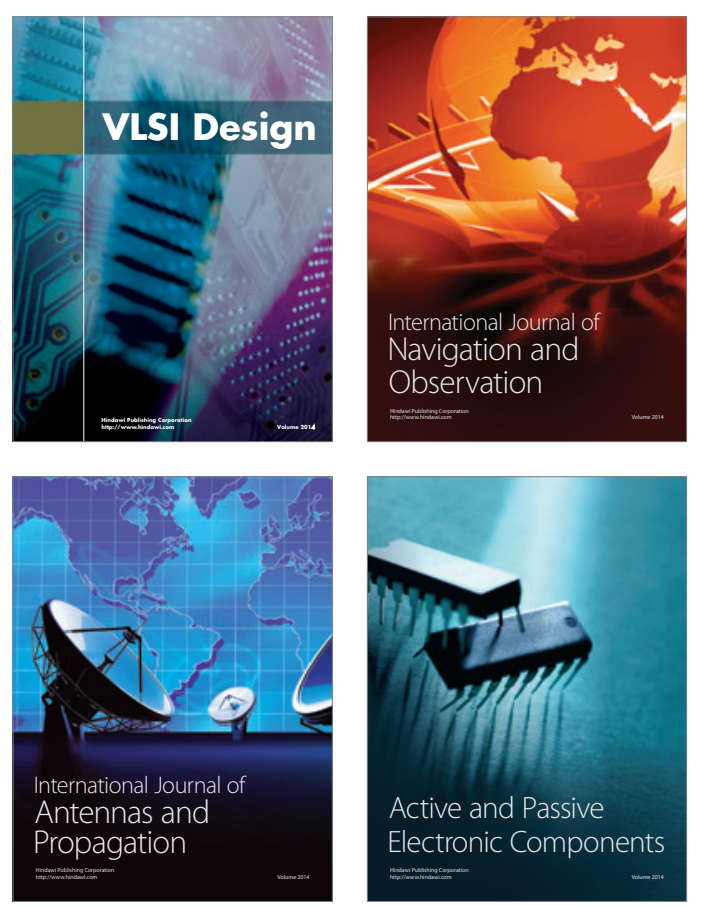
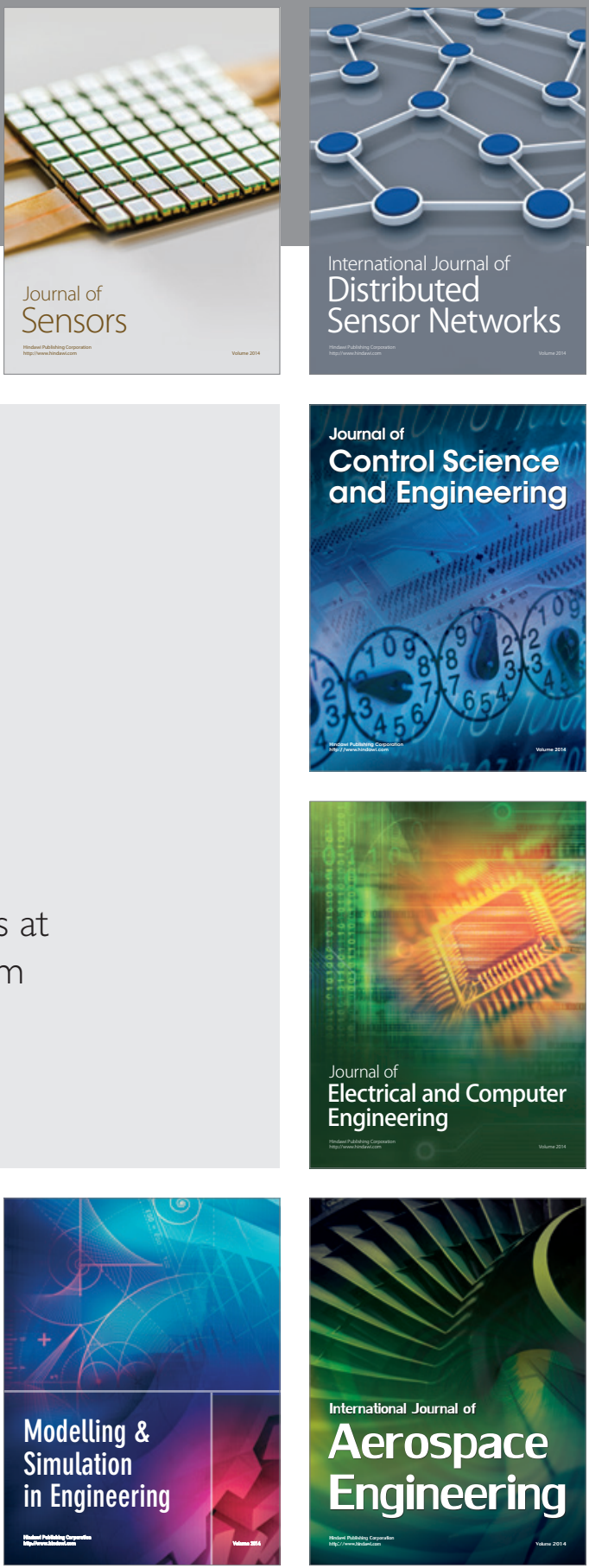

Journal of

Control Science

and Engineering
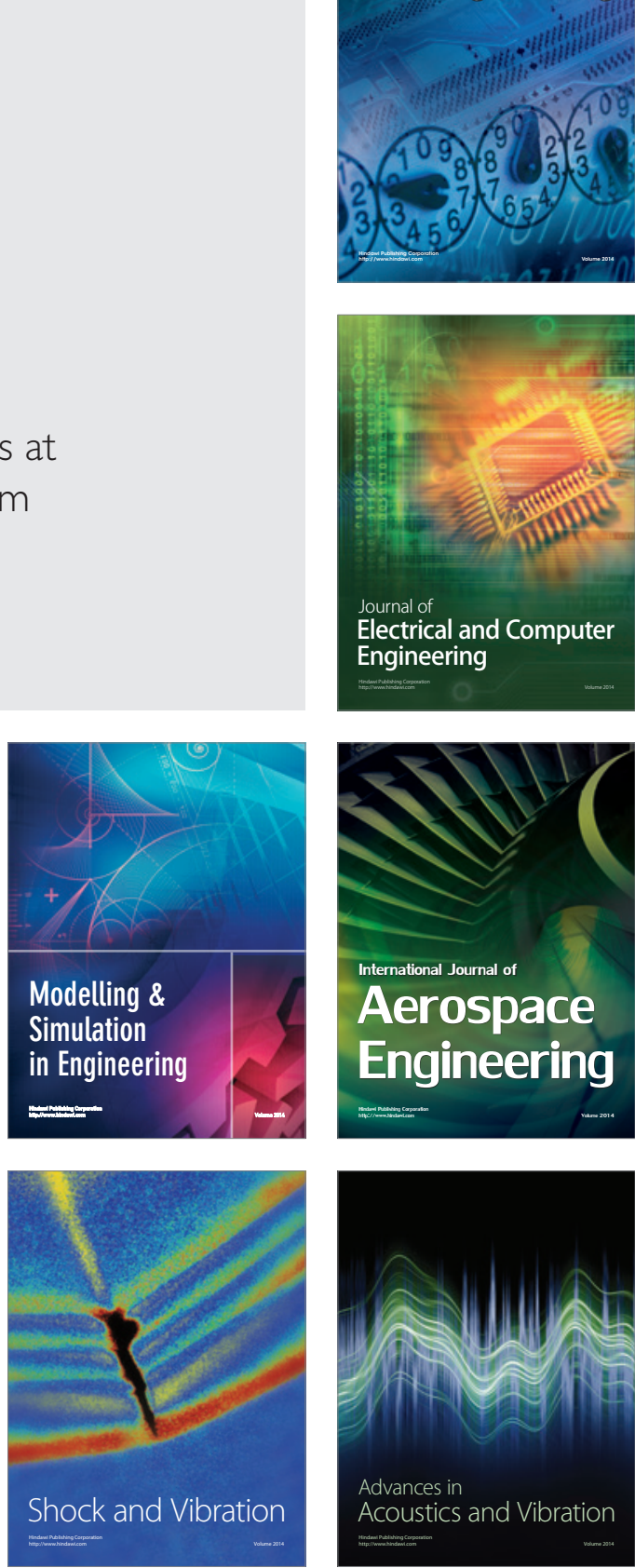